\title{
Advances in Our Understanding of Hyperthyroidism-associated Bone Loss
}

\author{
a report by \\ Mary Jane Brassill' and Graham R Williams²
}

\author{
1. Specialist Registrar, Department of Endocrinology, Cork University Hospital; 2. Professor of Endocrinology, and \\ Head, Molecular Endocrinology Group, Division of Medicine and MRC Clinical Sciences Centre, Imperial College London, Hammersmith Hospital
}

DOI:10.17925/EE.2008.04.02.103

Osteoporosis is characterised by low bone mass and deterioration of bone micro-architecture associated with increased bone fragility and susceptibility to fracture. In 2005 the incidence of osteoporotic fracture in the US was over two million, at a cost of nearly US $\$ 17$ billion. By 2025 , annual fractures and costs are projected to rise by almost $50 \%$, making this a major healthcare priority. ${ }^{1}$ Hyperthyroidism is an important cause of secondary osteoporosis ${ }^{2}$ and the relationship between the hypothalamic-pituitarythyroid (HPT) axis and bone is an important factor to consider in the management of sub-clinical hyperthyroidism and differentiated thyroid cancer. This article discusses animal models that provide insight into the underlying mechanisms that result in hyperthyroidism-associated bone loss. It will then review the clinical data investigating effects of hyperthyroidism on the skeleton.

Release of thyrotropin-releasing hormone (TRH) from the hypothalamus stimulates anterior pituitary thyrotrophs to secrete thyrotropin (TSH). TSH stimulates thyroid follicular cell proliferation and the synthesis and release of the thyroid hormones T4 (thyroxine) and T3 (3,5,3'-L-triiodothyronine). Synthesis and secretion of TRH and TSH are inhibited by direct negative feedback actions of thyroid hormones in the hypothalamus and pituitary gland. The HPT axis maintains a constant physiological reciprocal relationship between thyroid hormones and $\mathrm{TSH}^{3}$ The thyroid gland secretes approximately 10 -fold more of the pro-hormone $\mathrm{T} 4$ than its active metabolite T3. Most of the circulating T3 is formed in peripheral tissues via 5'-de-iodination of T4, a reaction performed by the type 1 and type 2 iodothyronine deiodinase enzymes, D1 and D2. Circulating thyroid hormones enter target cells by active transport mediated by several specific transporter proteins, of which monocarboxylate transporter-8 (MCT-8) is the best characterised. Once inside the cell, T4 can be activated locally to T3 by the D2 enzyme or, alternatively, T3 and T4 may be inactivated irreversibly by a third deiodinase enzyme, D3, which catalyses removal of the 5-iodine atom. The balance between activities of D2 and D3 enzymes within the target cell determines the amount of T3 available to the nuclear thyroid hormone receptors (TRs), which function as hormone-inducible transcription factors. ${ }^{4}$ Several TR isoforms have been described. TR $\beta 2$ is expressed primarily in the hypothalamus and pituitary gland, where it mediates negative feedback inhibition of TRH and TSH synthesis. TR $\alpha 1$ and TR $\beta 1$ are widely distributed in most tissues, but their relative levels of expression vary in temporo-spatial-specific patterns. Data from mutant mice suggest that $T R \alpha 1$ is the functionally predominant isoform expressed in the skeleton. ${ }^{4}$

Adult bone is a dynamic tissue that undergoes continuous re-modelling, a process mediated by the coupled activities of bone-resorbing osteoclasts and bone-forming osteoblasts. ${ }^{5}$ Precursors from the monocyte/macrophage cell lineage are activated and differentiate to mature bone-resorbing osteoclasts under the influence of macrophage colony-stimulating factor (MCS-F) and receptor activator of nuclear factor $\kappa \mathrm{B}$ ligand (RANKL). RANKL and osteoprotegerin (OPG), a decoy receptor for RANKL, are secreted by osteoblasts and function as critical regulators of osteoclast differentiation, acting via a local paracrine pathway that facilitates communication between the two cell lineages. The bone re-modelling cycle consists of three consecutive phases. In the resorption phase, mature, differentiated osteoclasts adhere to the bone surface and remove mineral and bone matrix through secretion of digestive lysosomal enzymes. ${ }^{6}$ Once resorption is complete there is a reversal phase in which osteoclasts undergo programmed cell death and mononuclear cells are seen on the bone surface. These cells may play a role in preparing the surface for osteoblasts to lay down new matrix and begin bone mineralisation, which occurs in the final formation phase. ${ }^{7}$

It is recognised that thyroid hormones play an essential role in both skeletal development and the maintenance of adult bone mass. Juvenile-acquired hypothyroidism leads to growth arrest, delayed bone maturation and short stature. Despite treatment, hypothyroidism in children can result in a permanent height deficit related to the duration of thyroxine deficiency before hormone replacement. ${ }^{8}$ In contrast, childhood thyrotoxicosis causes accelerated growth and bone development, but paradoxically also results in short stature due to premature closure of the epiphyseal growth plates. ${ }^{9}$ Changes in thyroid status in adults affect the bone re-modelling cycle. Hypothyroidism reduces bone turnover and prolongs the re-modelling cycle, ${ }^{10}$ and increased mineralisation in the formation phase can lead to elevated bone mineral density. Hyperthyroidism increases bone turnover, with increases in both osteoclast and osteoblast activities. ${ }^{10}$ As a result, the bone re-modelling cycle is shortened, although all phases of the cycle are not affected equally. While the duration of the resorption phase is largely unaltered, the duration of the formation phase is reduced significantly.

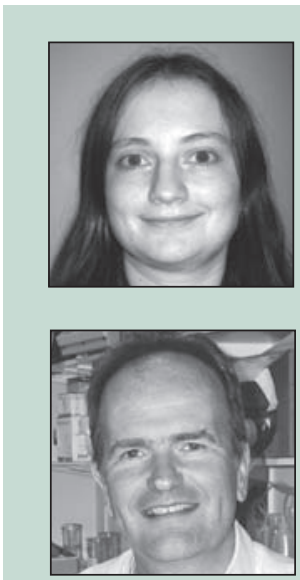

Mary Jane Brassill is a specialist registrar in Endocrinology at Cork University Hospital, a tertiary referral centre for endocrinology and diabetes in Southern Ireland. Dr Brassill is a member of the Irish Endocrinology Society and the British Society for Endocrinology. thyroid hormone action in bone. He is a member of the British and European Thyroid Association Executive Committees and a Council Member of the Society for Endocrinology.

E: graham.williams@imperial.ac.uk 
This leads to a failure to replace resorbed bone completely, resulting in net loss of about $10 \%$ of mineralised bone per cycle. ${ }^{4}$ Hyperthyroidismassociated bone loss has long been attributed to elevated levels of thyroid hormones. ${ }^{6}$ However, more recently a role for TSH as a direct inhibitor of bone turnover has been suggested by studies analysing skeletal abnormalities in TSH receptor knockout mice. ${ }^{11}$ The relative importance of thyroid hormones and TSH in the physiological control of bone turnover remains controversial. This is because the normal reciprocal relationship between thyroid hormones and TSH, which is maintained by the HPT axis, makes it difficult to separate the opposing skeletal effects of thyroid hormones and TSH in vivo, and further studies are required.

\section{Mechanisms of Bone Loss in Hyperthyroidism}

A number of studies in genetically modified mice have advanced our understanding of the actions of thyroid hormones on bone and the mechanisms of accelerated bone loss in hyperthyroidism. TR $\alpha^{0 / 0}$ mice, which lack all TR $\alpha$ isoforms, are euthyroid with normal T4 and TSH levels. Juveniles display a hypothyroid skeletal phenotype with growth retardation, delayed endochondral ossification and impaired mineralisation. Adult mice display increased trabecular bone mass with reduced bone resorption surfaces and reduced osteoclast numbers. ${ }^{12}$ Further studies on mice with dominant negative TR 1 mutations in different genetic backgrounds (TR $\alpha 1^{\mathrm{PV} /+}$ and TR $\alpha 1^{\mathrm{R} 384 \mathrm{C} /+}$ ) replicate these findings, although mice expressing these dominant-negative mutant receptors display more severe skeletal phenotypes than mice harbouring a deletion of the gene encoding TR $\alpha .{ }^{14,15}$ In contrast, TR $\beta^{-/-}$mice, which lack all TR $\beta$ isoforms, have a resistance to thyroid hormone with elevated T4 and TSH levels. Juveniles have advanced ossification and increased bone mineral deposition. Adult mice display an osteoporotic phenotype with reduced trabecular and cortical bone of lower mineralisation density, increased resorption surfaces and increased numbers of osteoclasts. ${ }^{12}$ Mice with a dominant negative mutation of TR $\beta$ in a different genetic background (TR $\beta^{\mathrm{PV} / \mathrm{PV}}$ ) also display a thyrotoxic skeletal phenotype with advanced ossification, premature closure of the growth plates and short stature, although the phenotype is more severe than observed in TR $\beta^{-/}$ mice. ${ }^{16}$ These findings are consistent with observations that TR $\alpha$ is the main functional TR isoform expressed in bone, whereas TR $\beta$ predominates in the pituitary gland. In TR $\alpha$ mutants a hypothyroid skeletal phenotype is present despite normal euthyroid status because T3 action in bone cells is impaired. TR $\beta$ mutants have elevated thyroid hormone and TSH levels due to disruption of the HPT axis. Skeletal thyrotoxicosis results due to increased TR $\alpha$ activity stimulated by the elevated circulating T3 levels. ${ }^{17}$ T3 regulates osteoblast proliferation and function directly, but it is currently unclear whether osteoclast proliferation and function is regulated directly by $\mathrm{T3}$ or via osteoblast-mediated effects. ${ }^{3}$

While these findings suggest that skeletal hyperthyroidism results from elevated T3 levels rather than low TSH levels, a direct role for TSH as a negative regulator of bone turnover has been suggested. ${ }^{11}$ The TSH receptor (TSHR), a G-protein-coupled receptor, is expressed in several cell types and was shown to be expressed in osteoclasts and osteoblasts in vitro. ${ }^{11}$ TSHR knockout mice ( $\mathrm{TSHR}^{-/}$) have congenital hypothyroidism, with undetectable levels of thyroid hormone and grossly elevated TSH levels. ${ }^{11}$ The mice had evidence of high bone turnover osteoporosis, and the authors postulated that TSH directly inhibits bone re-modelling. However, it has been noted that thyroid hormone replacement was instituted in these mice at post-natal day 21 , although the normal maximum growth velocity in mice is seen at two weeks of age, when T3 and T4 levels reach their physiological peak. ${ }^{12}$ It is possible that the accelerated bone re-modelling noted in TSHR ${ }^{-/}$mice reflects 'catch-up' growth due to delayed thyroid hormone replacement. , 12 $^{12}$

In order to delineate the relative importance of T3 and TSH in bone further, the skeletal phenotypes of two mouse models of congenital hypothyroidism were compared. ${ }^{13}$ Pax8 null mice (Pax8-/) lack a transcription factor required for thyroid follicular development. Hyt/hyt mice have a TSHR loss-of-function mutation. Both have severe hypothyroidism and grossly elevated TSH levels, however, Pax8 ${ }^{-1-}$ mice express a fully functional, normal TSHR, whereas hyt/hyt mice have a non-functional receptor. If TSH exerts major effects on the skeleton, these mice should display opposing skeletal phenotypes. However, both Pax8-- and hythyt mice have a similar phenotype of growth retardation and delayed ossification consistent with hypothyroidism, suggesting that the skeletal consequences of congenital hypothyroidism are independent of the actions of TSH. In vitro studies of TSH effects on osteoblasts and osteoclasts in this study did not demonstrate a functional response of skeletal cells to TSH or TSH receptor-stimulating antibodies. ${ }^{13}$

In summary, data obtained from animal models suggest that skeletal responses to hyperthyroidism are mediated by T3 acting via TR $\alpha$. In contrast, if TSH exerts predominant actions to inhibit bone turnover, the high concentrations of circulating TSH receptor-stimulating antibodies in Graves' disease should protect patients from bone loss. However, individuals with Graves' thyrotoxicosis are susceptible to osteoporosis and fracture, further suggesting that thyroid hormone excess is responsible for bone loss. ${ }^{18}$ Nevertheless, the field remains controversial. Recent studies have shown that administration of TSH to euthyroid, ovariectomised rats prevents bone loss or may even restore bone if TSH treatment is delayed until ovariectomy-induced bone loss has occurred. ${ }^{19} \mathrm{~A}$ recent suggestion is that TSH may act as a finetuning regulator of bone turnover in euthyroid individuals, particularly in conditions of high bone turnover such as oestrogen deficiency. ${ }^{3}$

\section{Clinical Data on Hyperthyroidism, Bone Loss and Risk of Fracture}

Our understanding of hyperthyroidism-associated bone loss has also been advanced by clinical studies. It is clear that untreated hyperthyroidism significantly decreases bone mineral density (BMD) and increases the risk of osteoporotic fractures. ${ }^{18}$ Several studies have also investigated BMD and fracture risk in patients previously treated for hyperthyroidism. Many studies have demonstrated improvement in BMD following treatment of hyperthyroidism. ${ }^{20-23}$ Even though the length of follow-up and degree of improvement in BMD varied between studies, BMD did not return to normal in any of them. Despite this, a cross-sectional study of 164 women found BMD to be significantly reduced in patients in the first thee years following diagnosis of thyrotoxicosis. ${ }^{24}$ After this interval, however, BMD did not differ significantly compared with control subjects. It is clear that BMD improves after treatment of hyperthyroidism, although there are conflicting data regarding whether recovery is full following restoration of euthyroidism.

A more important clinical end-point to consider is the risk of fracture in patients with hyperthyroidism. Studies published in the 1990s revealed an association between hyperthyroidism and an increased risk of hip 25,26 and foot $^{27}$ fracture in post-menopausal women. Only one study demonstrated no increased risk of fracture in women with a history of hyperthyroidism; however, individuals in that study were found to have an earlier occurrence of first fracture compared with normal subjects with no prior history of thyrotoxicosis. ${ }^{28}$ A large retrospective, population-based study of 7,209 patients treated with radioactive iodine for hyperthyroidism demonstrated an 
increased risk of death due to fracture of the femur, with a 2.9-fold increased standardised mortality ratio. ${ }^{29}$ More recently, two large casecontrol studies have shown an increased risk of fracture in patients previously treated for hyperthyroidism. The first evaluated 11,776 patients previously treated for hyperthyroidism and found that fracture risk was increased around the time of diagnosis (incidence rate ratio 1.16-2.29), but decreased to normal after the diagnosis of hyperthyroidism was made. ${ }^{30}$ This study also noted that individuals treated with thyroidectomy had a significantly lower risk of fracture after diagnosis (relative risk $0.55-0.78$ ). A second study by the same group evaluated 124,655 consecutive patients with fractures ${ }^{31}$ in comparison with three age- and sex-matched randomised controls from the Danish population. An increased fracture risk was demonstrated in subjects in the five years following a diagnosis of hyperthyroidism. Use of antithyroid drugs was associated with a significant reduction in fracture risk, presumed to result from reduced thyroid hormone levels. ${ }^{31}$

Several studies have shown reduced BMD and increased fracture risk in patients with hyperthyroidism. Hyperthyroidism should be considered an established risk factor for secondary osteoporosis in clinical practice. Treatment results in an improvement in BMD and there is some evidence that the increased risk of fracture may be reversible, 30,31 although this is debatable as several population studies have demonstrated a prior history of thyrotoxicosis to be associated with an increased risk of fracture. ${ }^{26,27}$

\section{Sub-clinical Hyperthyroidism}

Sub-clinical hyperthyroidism is a biochemical abnormality defined as a TSH level below the normal reference range in the presence of normal circulating T4 and T3 levels. It most commonly occurs in elderly individuals in association with multinodular goitre. Several studies have shown decreased BMD at various sites in post-menopausal women with untreated sub-clinical hyperthyroidism, including femoral neck, ${ }^{32-35}$ lumbar spine, ${ }^{32,33}$ radius $^{35}$ and phalanges. ${ }^{33}$ Further studies corroborate this evidence and also suggest that BMD may recover following treatment. ${ }^{36,37}$ A prospective, non-randomised study evaluated BMD in post-menopausal women with sub-clinical hyperthyroidism. ${ }^{36}$ Sixteen women were treated with radioactive iodine and compared with 12 untreated controls. The treated group had increased $\mathrm{BMD}$ at the hip and spine after two years. The untreated group experienced a $2 \%$ per year decrease in bone mass. A further prospective study of 16 post-menopausal women with sub-clinical hyperthyroidism and multinodular goitre showed an improvement in distal forearm BMD over two years in the eight subjects treated with methimazole. ${ }^{37}$

There are fewer data on the effect of sub-clinical hyperthyroidism on BMD in pre-menopausal women, although a few studies have shown no effect. $32,35,38$ One study showed a decrease in BMD in femoral neck and phalanges but not lumbar spine. ${ }^{33}$ The effect of sub-clinical hyperthyroidism on BMD in men has not been investigated adequately. However, a recent study showed a significant reduction in BMD at the ultra-distal forearm in men with a TSH level below the 25th percentile. ${ }^{39}$

Post-menopausal women with endogenous sub-clinical hyperthyroidism appear to be at risk of bone loss, most consistently in the femoral neck, but also in the lumbar spine. Most data suggest that pre-menopausal women do not share this risk. While the risk of fracture due to sub-clinical hyperthyroidism is less well defined, a prospective cohort study in postmenopausal women addressed this issue. ${ }^{40}$ The authors showed that a suppressed TSH, from any cause, led to a 3.6-fold increased risk of hip fracture and a 4.5 -fold increased risk of vertebral fracture.
A similar scenario was also seen in studies analysing the effect on BMD of suppressive doses of thyroxine in the treatment of differentiated thyroid cancer. A recent systematic review selected and analysed 21 studies. ${ }^{41}$ The authors concluded that while no increased risk was seen in men or pre-menopausal women, post-menopausal women on suppressive doses of thyroxine were at risk of reduced bone density. This conclusion correlated with the findings of previous reviews. ${ }^{6,42}$ It is recommended that post-menopausal women with thyroid cancer at high risk of recurrence, who require long-term TSH suppression with thyroxine, be monitored closely for osteoporosis. ${ }^{43}$

\section{Role of Thyroid-stimulating Hormone in} Hyperthyroidism-associated Bone Loss

Studies in animal models led to the proposal that hyperthyroidism-associated bone loss may result from TSH deficiency, with TSH acting as a direct inhibitor of bone turnover. ${ }^{11}$ Some authors have postulated that the findings in endogenous sub-clinical hyperthyroidism and exogenous TSH suppression suggest a critical role for TSH over thyroid hormone in hyperthyroidismassociated bone loss. ${ }^{44}$ Two cross-sectional studies have also shown a linear association between TSH levels and BMD in post-menopausal women. ${ }^{45,46} \mathrm{~A}$ population-based study of nearly 2,000 men and post-menopausal women found no association between BMD and TSH in normal individuals with TSH levels within the population reference range. ${ }^{39}$ Another study found that while TSH levels correlated inversely with markers of bone turnover in thyroid cancer patients treated with thyroxine replacement, they did not correlate with BMD. In this study a positive association between T4 and BMD was identified. ${ }^{47}$ In all of these clinical and population studies, it is important to note that the physiological reciprocal relationship between TSH and thyroid hormone is maintained by the HPT axis.

A number of clinical scenarios in which this reciprocal relationship is disrupted can be useful when considering the role of TSH in the skeletal manifestations of hyperthyroidism. The most common of these is Graves' disease, in which TSH receptor-stimulating antibodies are present, leading to persistent activation of the TSH receptor and increased thyroid hormone levels. However, there is no evidence that persistent stimulation of TSH receptor in Graves' disease protects against bone loss as patients with the disease are at an increased risk of osteoporotic fracture. ${ }^{30}$ Resistance to thyroid hormone (RTH) results from a dominant negative mutation of TR $\beta$ leading to elevated levels of TSH and thyroid hormone, as described above in TR $\beta$ mutant mice. ${ }^{15}$ In humans, RTH results in a variable mixture of features of hypo- and hyperthyroidism, with inconsistent clinical presentations. ${ }^{48}$ Some studies have observed increased bone turnover and short stature, ${ }^{49,50}$ features that are consistent with skeletal thyrotoxicosis observed in TR $\beta$ mutant mice.

Isolated TSH deficiency is a severe form of congenital hypothyroidism that results from a loss of function mutation of the TSH $\beta$-subunit. A recent study analysed two male siblings 10 and seven years of age with this disorder. ${ }^{51}$ The brothers both had undetectable TSH levels but normal thyroid hormone levels following thyroxine replacement. BMD and markers of bone metabolism were within the normal range in both patients, suggesting that a lifetime of TSH deficiency had no adverse effects on BMD. ${ }^{51}$

Following on from studies showing an increase in BMD in ovariectomised rats following TSH administration, ${ }^{19}$ a few clinical studies evaluating the skeletal effects of TSH administration in thyroid cancer patients have been published. Results from these studies have been conflicting. One study 
demonstrated a fall in type 1 collagen C-telopeptides (a marker of bone resorption) and an increase in bone alkaline phosphatase (a marker of bone formation) in post-menopausal women following administration of recombinant human TSH (rhTSH). 52 The authors suggested that the findings indicate decreased bone turnover in these patients, although effects were absent in pre-menopausal women and men. Similarly, no changes in levels of serum OPG, which inhibits osteoclastogenesis by binding to RANKL, were observed in response to TSH. ${ }^{52}$

A second study also found that administration of rhTSH had no effect on serum OPG or RANKL concentrations. ${ }^{53}$ In contrast to the first study, no changes in biochemical markers of osteoclastic bone resorption were observed. The results of a third study showed an increase in serum $\mathrm{N}$-terminal propeptide of type-I procollagen (PINP), a marker of bone formation, in post-menopausal women. ${ }^{53}$ This study also demonstrated increased RANKL concentrations in men and post-menopausal women treated with TSH, but no change in OPG levels were observed. No consensus can be drawn from the limited data available and further studies are required to investigate the possible skeletal effects of rhTSH administration.

\section{Summary}

Increased bone turnover in hyperthyroidism results in loss of mineralised bone and osteoporosis. Studies on animal models suggest that T3, acting via $T R \alpha$, is responsible for skeletal responses to hyperthyroidism. While TSHR is expressed in skeletal cells, the role of TSH in hyperthyroidismassociated bone loss remains controversial. Clinical conditions in which the HPT axis is disrupted do not suggest a direct role for TSH. Administration of TSH was shown to prevent, and possibly restore, bone loss in oestrogen-deficient rodents. Clinical studies of rhTSH administration are limited and the results are conflicting. A potential explanation is a role for TSH as a 'fine-tuning' regulator of bone turnover in euthyroid individuals in conditions of high bone turnover. Clinical studies have shown reduced BMD and increased risk of fracture in patients with hyperthyroidism. Evidence is conflicting as to whether this increased risk is reversible following treatment. Post-menopausal women with endogenous sub-clinical hyperthyroidism and those with thyroid cancer taking suppressive doses of thyroxine are also at risk of reduced $\mathrm{BMD}$ and fracture and should be appropriately assessed and monitored. There are insufficient data to confirm an increased risk in pre-menopausal women, or men, with these conditions at present.
1. Burge R, Dawson-Hughes B, Solomon DH, et al., Incidence and economic burden of osteoporosis-related fractures in the United States, 2005-2025, J Bone Miner Res, 2007;22(3):465-75.

2. Mosekilde L, Eriksen EF, Charles P, Effects of thyroid hormones on bone and mineral metabolism, Endocrinol Metab Clin North Am, 1990;19(1):35-63.

3. Basset JH, Williams GR, Critical role of the hypothalamic-pituitarythyroid axis in bone, Bone, 2008:43:418-26.

4. Bassett JH, Williams GR, The molecular actions of thyroid hormone in bone, Trends Endocrinol Metab, 2003;14:356-64.

5. Wexler JA, Sharretts J, Thyroid and bone, Endocinol Metab Clin North Am, 2007;36(3):673-705.

6. Murphy E, Williams GR, The thyroid and the skeleton, Clin Endocrinol, 2004;61:285-98.

7. Hadjidakis DJ, Androulakis II, Bone remodeling, Ann N Y Acad Sci, 2006;1092:385-96.

8. Rivkees $\mathrm{SA}$, Bode $\mathrm{HH}$, Crawford JD, Long-term growth in juvenile acquired hypothyroidism: the failure to achieve normal adult stature, N Eng/ J Med, 1988;318(10):599-602.

9. Segni M, Gorman CA, The aftermath of childhood hyperthyroidism, J Pediatr Endocrinol Metab, 2001;14:1277-82.

10. Altabas V, Berkovic $M$, Becejac $B$, Solter $M$, Bone re-modeling and thyroid function, Acta Clin Croat, 2007:46:41-7.

11. Abe E, Marians RC, Yu W, et al., TSH is a negative regulator of skeletal remodeling, Cell, 2003;115:151-62.

12. Bassett JH, O'Shea PJ, Sriskantharajah S, et al., Thyroid hormone excess rather than thyrotropin deficiency induces osteoporosis in hyperthyroidism, Mol Endocrinol, 2007;21(5):1095-1107.

13. Bassett JH, et al., A lack of thyroid hormones rather than excess thyrotropin causes abnormal skeletal development in hypothyroidism, Mol Endocrinol, 2008;22(2):501-12.

14. O'Shea PJ, Bassett JH, Sriskantharajah S, et al., Contrasting skeletal phenotypes in mice with an identical mutations targeted to thyroid hormone receptor $\alpha 1$ or $\beta$, Mol Endocrinol, 2005; 19(12):3045-59.

15. Bassett JH, Nordstrom K, Boyde A, et al., Thyroid status during skeletal development determines adult bone structure and mineralization, Mol Endocrinol, 2007;21(8):1893-1904.

16. O'Shea PJ, Harvey CB, Suzuki H, et al., A thyrotoxic skeletal phenotype of advanced bone formation in mice with resistance to thyroid hormone, Mol Endocrinol, 2003;17(7):1410-24.

17. O'Shea PJ, et al., Characterisation of skeletal phenotypes of $T R \alpha 1^{P V}$ and TR $\beta^{P V}$ mutant mice: implications for tissue thyroid status, Nucl Recept Signal, 2006;4:e011.

18. Vestergaard $P$, Mosekilde L, Hyperthyroidism, bone mineral, and fracture risk - a meta-analysis, Thyroid, 2003;13(6):585-93.

19. Sampath TK, Simic P, Sendak R, et al., Thyroid-stimulating hormone restores bone volume, microarchitecture, and strength in ages ovariectomised rats, J Bone Miner Res, 2007;22(6):849-59.

20. Rosen C, Adler R, Longitudinal changes in lumbar bone density among thyrotoxic patients after attainment of euthyroidism, J Clin Endocrinol Metab, 1992;75(6):1531-4.

21. Diamond T, et al., Thyrotoxic bone disease in women: a potentially reversible disorder, Ann Intern Med, 1994;120(1):8-11.

22. Acotto CG, Niepomniszcze H, Vega E, et al., Ultrasound parameters and markers of bone turnover in hyperthyroidism: a longitudinal study, J Clin Densitom, 2004;7(2):201-8.

23. Grant $D$, McMurdo M, Mole P, et al., Is previous hyperthyroidism still a risk factor for osteoporosis in post-menopausal women? Clin Endocrinol (Oxf), 1995;43(3):339-45.

24. Karga H, Papapetrou P, Korakovoni A, et al., Bone mineral density in hyperthyroidism, Clin Endocrinol (0xf), 2004;61(4):466-72.

25. Wejda B, Hintze G, Katschinski B, et al., Hip fractures and the thyroid: a case-control study, J Intern Med, 1995;237(3):241-7.

26. Cummings SR, Nevitt MC, Browner WS, et al., Risk factors for hip fracture in white women. Study of Osteoporotic Fractures Research Group, N Engl I Med, 1995;332(12):767-73.

27. Seeley DG, Kelsey J, Jergas M, Nevitt MC, Predictors of ankle and foot fractures in older women. The Study of Osteoporotic Fractures Research Group, J Bone Miner Res, 1996;11(9):1347-55.

28. Solomon BL, et al., Prevalence of fractures in postmenopausal women with thyroid disease, Thyroid, 1993;3(1):17-23.

29. Franklyn JA, Maisonneuve P, Sheppard MC, et al., Mortality after the treatment of hyperthyroidism with radioactive iodine, $N$ Engl J Med, 1998;338(11):712-18.

30. Vestergaard V, Mosekilde L, Fractures in patients with hyperthyroidism: a nationwide follow-up study in 16,249 patients, Thyroid, 2002:12(5);411-19.

31. Vestergaard V, et al., Influence of hyper- and hypothyroidism, and the effects of treatment with antithyroid drugs and levothyroxine on fracture risk, Calcif Tissue Int, 2005;77(3):139-44.

32. Foldes J, Lakatos P, Zsadanyi J, Horvath C, Decreased serum IGFand dehydroepiandrosterone sulphate may be risk factors for the development of reduced bone mass in postmenopausal women with endogenous subclinical hyperthyroidism, Eur J Endocrinol, 1997;136(3):277-81

33. Tauchmanova L, Nuzzo V, Del Puente A, et al., Reduced bone mass detected by bone quantitative ultrasonometry and DEXA in pre- and postmenopausal women with endogenous subclinical hyperthyroidism, Maturitas, 2004;48(3):299-306.

34. Lee WY, Oh KW, Rhee EJ, et al., Relationship between subclinical thyroid dysfunction and femoral neck bone mineral density in women, Arch Med Res, 2006;37(4):511-16.

35. Foldes J, et al., Bone mineral density in patients with endogenous sub-clinical hyperthyroidism: is this thyroid status a risk factor for osteoporosis?, Clin Endocrinol (Oxf), 1993;39(5):521-7.

36. Faber J, Jensen I, Petersen L, et al., Normalisation of serum thyrotropin by means of radioactive iodine treatment in subclinical hyperthyroidism: effect on bone loss in post-menopausal women, Clin Endocrinol (Oxf), 1998;48(3):285-90.
37. Mudde AH, et al., Bone metabolism during anti-thyroid drug treatment of endogenous sub-clinical hyperthyroidism, Clin Endocrinol (0xf), 1994;41(4):421-4.

38. Gurlek A, Gedik 0 , Effect of endogenous sub-clinical hyperthyroidism on bone metabolism and bone mineral density in pre-menopausal women, Thyroid, 1999;9(6):539-43.

39. Grimnes $G$, et al., The relationship between serum TSH and bone mineral density in men and post-menopausal women: the Tromsø study, Thyroid, 2008;18(11):1147-55.

40. Bauer DC, Ettinger B, Nevitt MC, Stone KL, Risk for fracture in women with low serum levels of thyroid-stimulating hormone, Ann Intern Med, 2001;134(7):561-8.

41. Heemstra KA, et al., The effects of thyrotropin-suppressive therapy on bone metabolism in patients with well-differentiated thyroid carcinoma, Thyroid, 2006;16(6):583-91.

42. Quan ML, et al., Bone mineral density in well-differentiated thyroid cancer patients treated with suppressive thyroxine: a systematic overview of the literature, J Surg Oncol, 2002;79(1):62-9.

43. Biondi B, et al., Thyroid-hormone therapy and thyroid cancer: a reassessment, Nat Clin Pract Endocrinol Metab, 2005;1(1):32-40.

44. Sun L, Davies TF, Blair HC, et al., TSH and bone loss, Ann N Y Acad Sci, 2006;1068:309-18.

45. Kim DJ, Khang YH, Koh JM, et al., Low normal TSH levels are associated with low bone mineral density in healthy postmenopausal women, Clin Endocrinol (0xf), 2006;64(1):86-90.

46. Morris MS, The association between serum thyroid-stimulating hormone in its reference range and bone status in postmenopausal American women, Bone, 2007:40(4):1128-34.

47. Van der Deure WM, et al., Effects of serum TSH and FT4 levels and the TSHR-Asp727Glu polymorphism on bone: the Rotterdam Study, Clin Endocrinol (Oxf), 2008;68(2):175-81.

48. Weiss RE, et al., Treatment of resistance to thyroid hormoneprimum non nocere, J Clin Endocrinol Metab, 1999;84(2):401-4.

49. Weiss RE, Refetoff $S$, Effect of thyroid hormone on growth. Lessons from the syndrome of resistance to thyroid hormone, Endocrinol Metab Clin North Am, 1996;25(3):719-30.

50. Brucker-Davis F, et al., Genetic and clinical features of 42 kindreds with resistance to thyroid hormone. The National Institutes of Health Prospective Study, Ann Intern Med, 1995;123(8):572-83.

51. Papadimitrious A, Papadimitriou DT, Papadopoulou A, et al., Low TSH levels are not associated with osteoporosis in childhood, Eur J Endocrinol, 2007;157(2):221-3.

52. Giusti M, Cecoli F, Ghiara C, et al., Recombinant human thyroid stimulating hormone does not acutely change serum osteoprotegerin and soluble receptor activator of nuclear factorkappaBeta ligand in patients under evaluation for differentiated thyroid carcinoma, Hormones, 2007;6(4):304-13.

53. Martini G, Gennari L, De Paola V, et al., The effects of recombinant TSH on bone turnover markers and serum osteoprotegerin and RANKL levels, Thyroid, 2008;18(4):455-60. 